\title{
Centralized Control Center Implementation for Synergistic Operation of Distributed Multifunctional Single-Phase Grid-Tie Inverters in a Microgrid
}

\author{
Helmo Kelis Morales-Paredes ${ }^{(0)}$, Member, IEEE, Jakson Paulo Bonaldo, \\ and José Antenor Pomilio ${ }^{(}$, Senior Member, IEEE
}

\begin{abstract}
This study discusses a centralized and coordinated control of distributed multifunctional inverters connected to an electric grid. The multifunctional operation is based on the conservative power theory, which makes it possible to identify undesired current components that can be compensated. The inverters that make up the microgrid are managed through a transmission control protocol/internet protocol (TCP/IP) communication network, allowing commands from a control center, while also allowing monitoring and gauging of electrical quantities of interest related to each of the inverters. Using the control center, it is possible to adjust the current necessary for compensation, which can be shared among inverters in a coordinated manner and according to their nominal capacities. Adjustments are made by sending references to certain indexes for desired power quality at the coupling point of each inverter. Experiment results explore some compensation alternatives, employing communication flexibility among the devices that comprise the microgrid.
\end{abstract}

Index Terms-Conservative power theory (CPT), coordinated control, microgrids, multifunctional inverters, power quality (PQ).

\section{INTRODUCTION}

$\mathbf{O}$ VER the last decade, the electrical industry has made important inroads in the expansion of renewable energy sources (RESs). These advances contributed to the growth of

Manuscript received June 16, 2017; revised October 6, 2017 and December 18, 2017; accepted January 14, 2018. Date of publication February 5, 2018; date of current version June 1, 2018. This work was supported in part by the Sao Paulo Research Foundation (FAPESP) under Grant 2016/08645-9 and in part by the National Council for Scientific and Technological Development (CNPq) under Grant 424853/2016-7 and Grant 302257/2015-2. (Corresponding author: Helmo Kelis MoralesParedes.)

H. K. Morales-Paredes is with the Institute of Science and Technology, São Paulo State University (UNESP), Sorocaba 19014-020, Brazil (e-mail: hmorales@sorocaba.unesp.br).

J. P. Bonaldo is with the Federal University of Technology-Paraná, Campo Mourão 87301-899, Brazil (e-mail: jbonaldo@utfpr.edu.br).

J. A. Pomilio is with the School of Electrical and Computer Engineering, University of Campinas, Campinas 13083-970, Brazil (e-mail: antenor@dsce.fee.unicamp.br).

Color versions of one or more of the figures in this paper are available online at http://ieeexplore.ieee.org.

Digital Object Identifier 10.1109/TIE.2018.2801780 distributed generation [1]-[5] by applying information and communication technology in electrical systems [6], [7]. Thus, production, transport, distribution, and end use of electrical power have become more efficient, environmentally sustainable, reliable, and safer. However, distributed energy systems (DESs) changed the traditional one-directional flow of energy to a bidirectional flow, which increased the complexity of power dispatch. On the other hand, the nonlinear loads connected to the distribution system can cause power quality (PQ) problems. In particular, current and voltage harmonics are becoming important concerns [8].

Every aspect mentioned above can be addressed applying suitable control strategies for multifunctional grid-tie inverters (MFGTIs), which are able to, simultaneously, integrate RESs in DESs and perform compensation of PQ problems [9]-[16]. The combination of these abilities in single equipment contributes to reduce the installation and operation costs of DESs. This approach is especially consistent if the MFGTI becomes part of distribution management systems. Therefore, RES consisting of solar or wind power could be connected to the grid via MFGTIs, which often do not operate at full capacity due to the intermittent behavior of these power sources. The available volt-ampere (VA), not used to transfer power produced by RES to the grid, can be used for auxiliary services, considering a minimal increase in system implementation costs.

Initial distributed compensation strategies were based on the application of active power filters (APF) [17]-[21] and static VAR compensator cooperating with APFs [22], [23]. Within the present scenario, i.e., increased RES penetration, the use of MFGTIs may also contribute to expand possibilities for implementing strategies for coordinated control in DES. For example, the MFGTIs can be used for injecting active power into the distribution system, sharing reactive power, voltage regulation at points of common coupling (PCC), and harmonic compensation [24]-[32].

Recently, studies have begun to discuss the cooperative operation of multiple converters integrating a microgrid. However, due to implementation difficulties-among which converter communication, management, and supervision infrastructure are included - some of these studies have been restricted to 


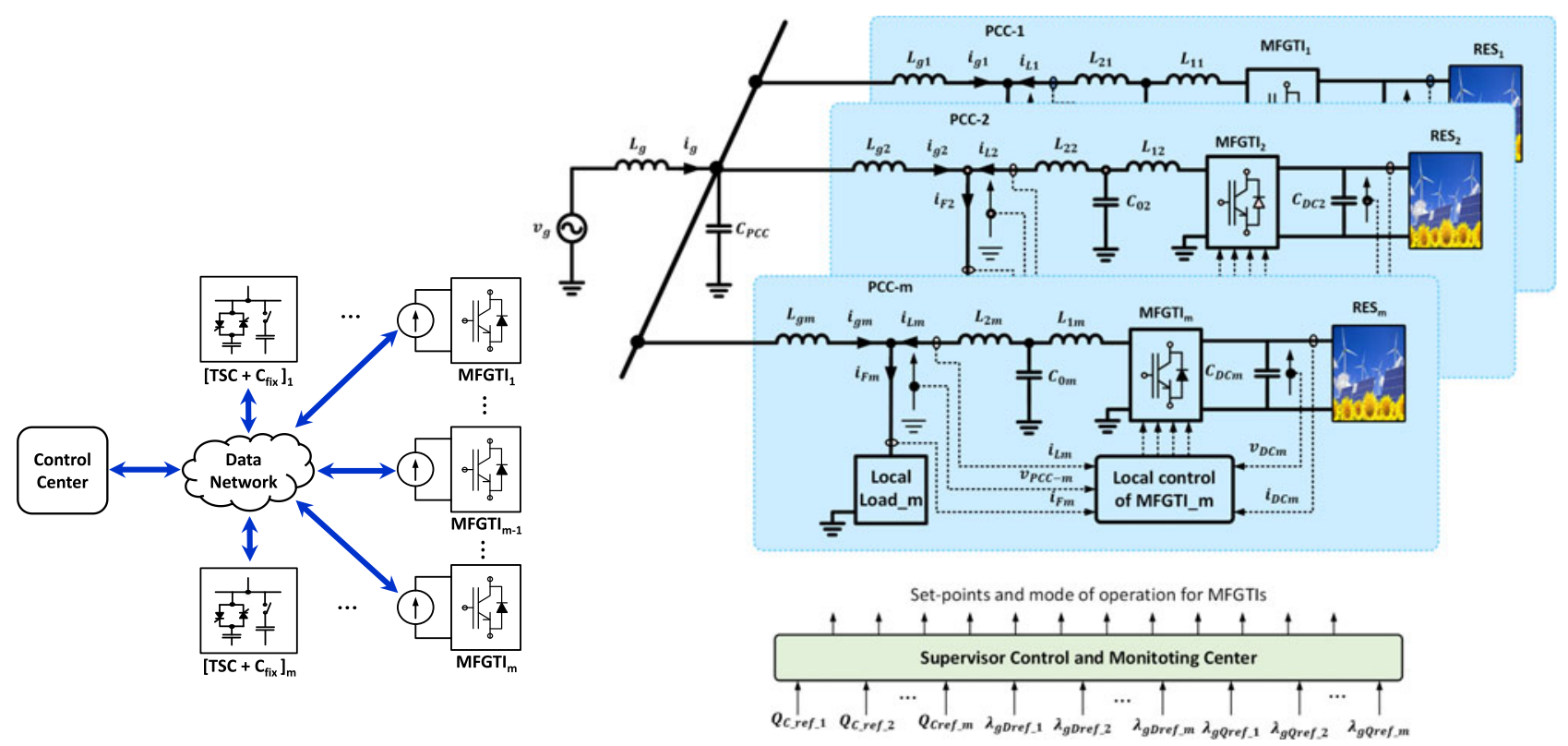

(a)

(b)

Fig. 1. Supervision and centralized control structure for MFGTIs and reactive compensators spread throughout a microgrid. (a) Overview of the communication structure. (b) Overall scheme of distributed MFGTIs.

simulation environments [27], [28]. One of the contributions of our research is to show a simple and effective way to implement control, management, and supervision infrastructure for multiple MFGTIs spread throughout a microgrid.

From the point of view of disturbance compensation, the proposed approach provides a different perspective of that discussed in [13] and [30], in which the MFGTI is controlled to achieve specific PQ requirement at a single PCC. Unlike [13] and [30], in our study, the control strategy is expanded to several MFGTIs considering a synergistic operation to regulate the active power, reactive energy/power, and harmonics synthesized by each MFGTIs. Thus, PQ requirement at PCC is globally achieve by coordinated operation of all MFGTIs. Moreover, the control approach is expanded to synergy operation with other compensators [i.e., capacitors banks or thyristor switched capacitors (TSC)].

The main objective of this study is to provide a platform that enables the coordinated operation of distributed MFGTIs. This platform is composed of a control center and MFGTIs with communication capability. The control center acts as centralized control and supervisory mode being able to remotely transmit and receive data to MFGTIs. Through this platform, the microgrid's operator can set and adjust parameters that define the functionality of each MFGTI. Using a communication network infrastructure [as shown in short in Fig. 1(a)], the control center establishes a two-way communication channel with each MFGTI, which in turn have the capacity to process and answer the received commands.

MFGTIs are able to receive remote commands from the centralized control center, such as reference for power factor at the PCC, reference for reactive power injection, and adjustment of the level of harmonic compensation. Therefore, MFGTIs are able to cooperate among each other and also with passive or ac- tive PQ compensators, such as TSC and capacitor banks. From a practical point of view, this approach is satisfactory for small microgrids (homes or commercial/industrial installations), which includes a range of local power sources.

A microgrid on laboratory scale [as shown in Fig. 1(b)] was created to validate the distributed operation of MFGTIs, as well as the coordinated communication and operation infrastructure. Finally, experimental results showed the validity and efficiency of the proposed approach to improve PQ by means of cooperation among MFGTIs and compensation elements in the microgrid.

\section{Modeling AND CONTROL OF MFGTI}

The local control for each MFGTI comprises two loops as discussed in [13]. The first one is a fast current loop to control the MFGTI current and the other is a slower voltage loop, responsible for maintaining constant dc bus voltage. An additional loop is used to control the current in the dc-dc converter, which is responsible for making the connection between the local source and the inverter's dc bus. For the sake of simplicity, the dc-dc converter and RES are treated as current sources $\left(I_{\mathrm{DC} m}\right)$.

The transfer function of the output current $\left(i_{F}\right)$ of the MFGTI is given by (1) as shown at the bottom of the next page and can be obtained by following the block diagram shown in Fig. 2. The dumping factor $\left(K_{D}\right)$ is the capacitor current feedback gain that contributes to the active damping of the $L C L$ filter [33], [34].

The open-loop transfer function $G_{\mathrm{OL}}(s)$ given by (2) is obtained considering time delay due to the pulse width modulation modulator and delay due to the digital implementation of the current controller (3) [35]. The antialiasing filter modeled by (4) is placed at the input of the analog-to-digital converter. 


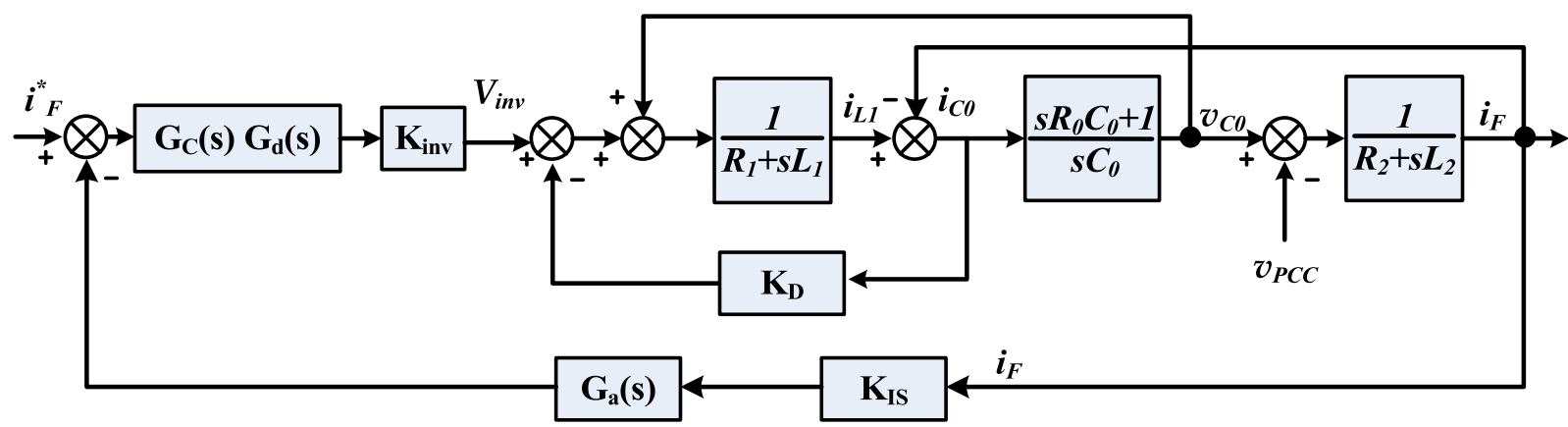

Fig. 2. Current loop block diagram of each MFGTI.

TABLE I

PARAMETERS OF THE CURRENT CONTROL LOOP

\begin{tabular}{lccc}
\hline \hline$K_{\text {inv }}$ & 300 & $K_{c}$ & 0.95 \\
$K_{\mathrm{IS}}$ & 0.0667 & $K_{I_{\mathrm{PR}}}$ & 100 \\
$\omega_{\mathrm{CL}}$ & $6.28[\mathrm{krad} / \mathrm{s}]$ & $\omega_{c_{\text {anti }}}$ & $62.8[\mathrm{krad} / \mathrm{s}]$ \\
$\omega_{c \mathrm{PR}}$ & $5[\mathrm{rad} / \mathrm{s}]$ & $\omega_{o}$ & $377[\mathrm{rad} / \mathrm{s}]$ \\
$T_{s}$ & $38[\mu \mathrm{s}]$ & $K_{D}$ & 0.5 \\
\hline
\end{tabular}

The inverter gain is given by $K_{\text {inv }}$, whereas $K_{\text {IS }}$ is the gain of the current sensor. The cutoff frequency of the antialiasing filter is $\omega_{c_{-} \text {anti }}$ and the sampling time is given by $T_{s}$, which is half the switching time

$$
\begin{aligned}
G_{\mathrm{OL}}(s) & =K_{\mathrm{inv}} K_{\mathrm{IS}} G_{\mathrm{FD}}(s) G_{d}(s) G_{a}(s) G_{C}(s) \\
G_{d}(s) & =\frac{1-s \frac{1}{2} T_{s}}{1+s \frac{1}{2} T_{s}} \\
G_{a}(s) & =\frac{\omega_{c_{\_} \text {anti }}}{s+\omega_{c_{\_} \text {anti }}} .
\end{aligned}
$$

The current controller is of proportional plus resonant harmonic type [36] and its transfer function is given by

$$
G_{C}(s)=K_{C}+\sum_{h=1,3,5, \ldots, 15} \frac{2 K_{I_{\mathrm{PR}}} \omega_{c_{\mathrm{PR}}} s}{s^{2}+2 \omega_{c_{\mathrm{PR}}} s+\left(h \omega_{0}\right)^{2}}
$$

The current controller was designed using the open-loop transfer function (2). The bandwidth of the current loop was set at $1.2 \mathrm{kHz}$ aiming the compensation of current harmonics. In addition, the loop gain at harmonics frequencies is increased using the resonant controller (5) through the adjustment of the resonant peaks by $K_{\mathrm{IPR}}$. In order to grant the system stability and allowing a fast transient response, the phase margin was set to $45^{\circ}$ with a gain margin of $6 \mathrm{~dB}$. The parameters of the current control loop are summarized in Table I.

On the other hand, Fig. 3 shows the block diagram of the bus voltage control loop. The transfer function $G_{\mathrm{DC}}(s)$, given

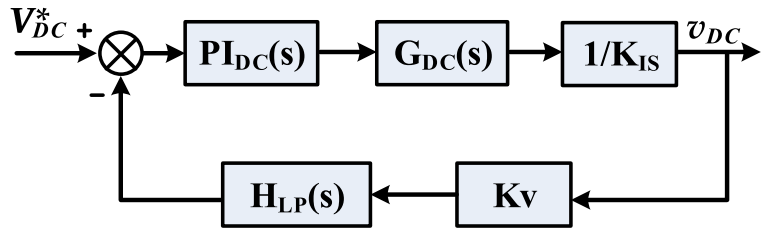

Fig. 3. Block diagram of the dc voltage control loop.

in (6), is obtained by a small signal analysis. It establishes a relationship between the dc bus voltage $\left(v_{\mathrm{DC}}\right)$ and the current peak at the inverter output, i.e., the current control variable $i_{G_{-} p}$. $V_{\text {PCC_rms }}$ stands for the rms value of grid voltage $(127 \mathrm{~V})$, whereas $V_{\mathrm{DC}}$ is the average value of the dc bus voltage $(300 \mathrm{~V})$ and $C_{\mathrm{DC}}$ is the dc bus capacitance $(4.5 \mathrm{mF})$.

The low-pass filter, $H_{\mathrm{LP}}(s)$, in the feedback path is designed to attenuate the $120 \mathrm{~Hz}$ ripple present in the $v_{\mathrm{DC}}$ voltage. In this study, the cutoff frequency $\left(\omega_{c_{\mathrm{LP}}}\right)$ of the low-pass filter was set at $30 \mathrm{~Hz}$. The remaining parameters are as follows: the gain of the voltage sensor is $K_{V}=0.00333$ and $1 / K_{\text {IS }}$ is the resulting gain of the current control loop [37]. The transfer function of the proportional integral controller used to regulate the dc bus voltage is given by (7).

It is worth to note that the bandwidth of the dc bus voltage control loop should be narrow enough to avoid oscillation of $i_{G \_p}$ and mitigating the interaction of the dc voltage controller with the faster current loop, which has a higher bandwidth [35]. Therefore, the open-loop transfer function of the dc bus voltage control (8) is used to design $P I_{\mathrm{DC}}(s)$ aiming the achievement of a stable system with the bandwidth of $6 \mathrm{~Hz}$ and the phase margin of $70^{\circ}$. The parameters of the resulting PI controller are $K_{\mathrm{PDC}}=2.2$ and $K_{\mathrm{IDC}}=49$.

$$
G_{\mathrm{DC}}(s)=\frac{\tilde{v}_{\mathrm{DC}}(s)}{\tilde{i}_{G_{-} p}(s)}=\frac{V_{\mathrm{PCC} \_ \text {rms }}}{s \sqrt{2} C_{\mathrm{DC}} V_{\mathrm{DC}}}
$$

$$
G_{\mathrm{FD}}(s)=\frac{I_{F}(s)}{V_{\mathrm{inv}}(s)}=\frac{s C_{0} R_{0}+1}{s^{3} L_{1} L_{2} C_{0}+s^{2} C_{0}\left(\begin{array}{c}
K_{D} L_{2}+L_{1} R_{2}+L_{2} R_{1}+ \\
+L_{1} R_{0}+L_{2} R_{0}
\end{array}\right)+s\left(\begin{array}{c}
K_{D} R_{2} C_{0}+L_{1}+L_{2}+R_{1} R_{2} C_{0}+ \\
+R_{1} R_{0} C_{0}+R_{2} R_{0} C_{0}
\end{array}\right)+R_{1}+R_{2}}
$$




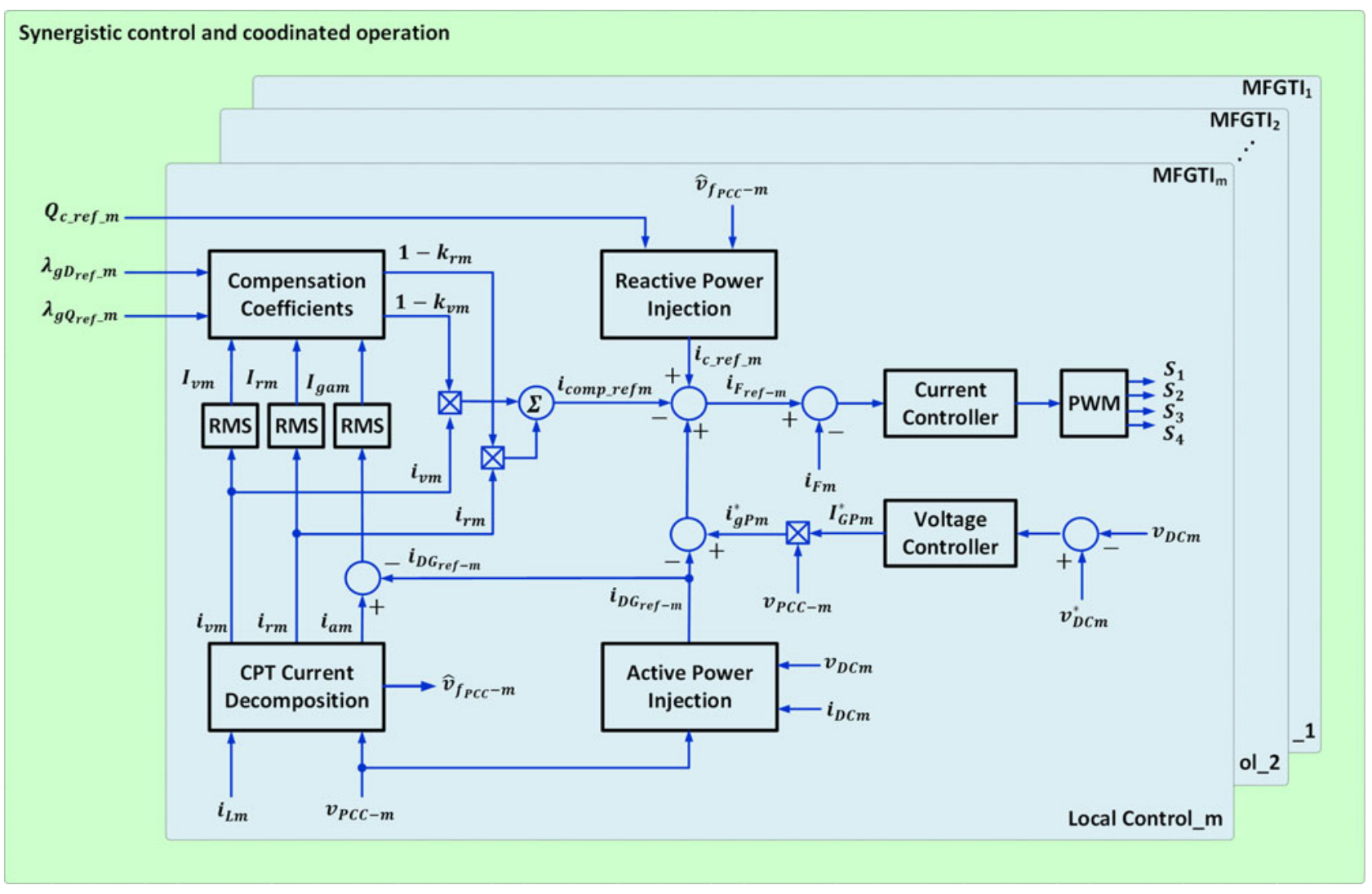

Fig. 4. Overview of the conceptual drawing of the synergistic flexible control with independent reactive power injection.

$$
\begin{aligned}
\mathrm{PI}_{\mathrm{DC}}(s) & =K_{\mathrm{PDC}}+\frac{K_{\mathrm{IDC}}}{s} \\
H_{\mathrm{DC}_{\mathrm{OL}}} & =\mathrm{PI}_{\mathrm{DC}}(s) G_{\mathrm{DC}}(s) H_{\mathrm{LP}}(s) K_{V} / K_{\mathrm{IS}} .
\end{aligned}
$$

\section{SYNERGISTIC CONTROL AND COOPERATIVE OPERATION STRATEGY}

In this section, the functionalities of the MFGTIs are discussed, among which the active and reactive power injection, harmonic mitigation, and compensation of reactive power due local loads are mentioned. Fig. 4 depicts the control diagram of each MFGTI.

These functionalities are integrated into the MFGTI by the generation of orthogonal (decoupled) current references, which are defined according to the conservative power theory (CPT) [29]. CPT current components have a forward relationship with the functionality intended to be implemented, i.e., harmonic content and reactive power, which makes the selection of the kind of disturbance to be compensated easier. Thus, beyond flexibility, selectivity, and voltage distortion immunity, the proposed method differs from others existing solutions for the fact that it does not need any kind of reference-frame transformation and synchronization algorithms.

Aiming at a better comprehension of this study, the main CPT definitions are presented below. Notice that the singlephase system is being considered. The lowercase letters stand for instantaneous signals, whereas the capital letters stand for rms values.

1) Active load current $\left(i_{a}\right)$ : responsible for transferring active power $\left(P=V I_{a}\right)$ from the source to the load.

2) Reactive load current $\left(i_{r}\right)$ : responsible for the reactive energy flow $W=\hat{V} I_{r}$, where $\hat{V}$ is the rms value of the unbiased integral of the load voltage [29]. This component may also be associated with reactive power through the ratio $\left(Q=V I_{r}\right)$.

3) Void (residual) load current $\left(i_{v}\right)$ : reflects nonlinearity between voltage and current, due mainly to the presence of switched devices. It does not transfer active power or reactive energy to the load. This portion of current is associated to void (residual) power, calculated through $\left(D=V I_{v}\right)$.

The apparent power $(A)$ is obtained through the product of respective rms voltage and rms current $(A=V I)$. Thus, the load power factor results in

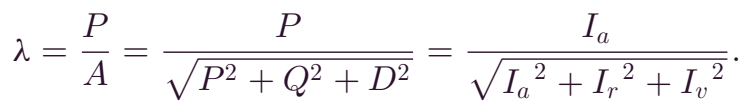

Furthermore, according to [13] and [31], the load power factor can also be decomposed into two decoupled factors, called load conformity factors. Each factor represents a specific load PQ disturbance. 
4) Reactivity factor: responsible for lagging between voltage and current

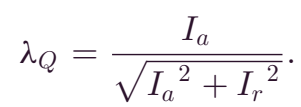

5) Distortion factor: responsible for nonlinearity between voltage and current

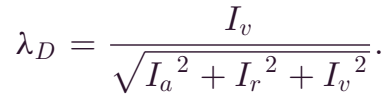

Based on these factors, Bonaldo et al. [13] discuss a technique for the flexible control of single-phase MFGTIs. This technique permits partial or total compensation of $i_{r}$ and $i_{v}$. The compensation reference signal is generated in the hope of improving the grid current waveform, to obtain specific results for conformity factors. Over the following sections, the technique proposed in [13] is expanded to make the coordinated control of MFGTIs distributed throughout a viable microgrid.

\section{A. Generation of the Reference Signal for the Synergistic and Coordinated Compensation of Unwanted Currents}

If the goal of the compensation is to guarantee that the grid shall only absorb or inject the active current, given by

$$
i_{a}=\frac{\left\langle v_{\mathrm{PCC}}, i\right\rangle}{V_{\mathrm{PCC}}^{2}} v_{\mathrm{PCC}}=\frac{P}{V_{\mathrm{PCC}}^{2}} v_{\mathrm{PCC}}
$$

where $v_{\mathrm{PCC}}$ and $i$ represent the instantaneous voltage and current gauged in the PCC, then the compensation circuit must absorb all the other current components, given by

$$
i_{\mathrm{comp}}=i_{a}-i=-\left(i_{r}+i_{v}\right) .
$$

The current reference for compensation $\left(i_{\text {comp }}\right)$ must be distributed to each of the microgrid components, including MFGTIs and other PQ compensation devices.

1) Reactive Power Injection: It is well-known that capacitors connected to the PCC can compensate reactive energy. However, MFGTIs may also contribute to the aim of implementing a fine adjustment of the reactive energy. To do so, the respective reference must include the reactive energy that should be injected by the converter.

The reactive injection reference $\left(Q_{c_{-} \text {ref }-m}\right)$ is sent remotely from the control center to each MFGTI. The reference for reactive injection (14) is calculated using the reactive power/energy concept [29]. The $\hat{v}_{f_{\mathrm{PCC}-m}}$ signal corresponds to the unbiased time integral of the fundamental component of PCC voltage [29] and $\hat{V}_{f_{\mathrm{PCC}}-m}$ represents the rms value of this voltage.

The equivalent susceptance $\left(B_{c_{\mathrm{ref}}-m}\right)$ and the fundamental reactive energy $\left(W_{f-m}\right)$ that corresponds to the reactive power $\left(Q_{c_{-} \text {ref-m }}\right)$ are calculated according to (15) and (16), where the angular frequency of the grid is $\omega$ and $V_{f_{\mathrm{PCC}}-m}=\omega \hat{V}_{f_{\mathrm{PCC}}-m}$ is the fundamental rms voltage at PCC

$$
\begin{aligned}
i_{c_{\mathrm{ref}-m}} & =\frac{W_{f-m}}{\hat{V}_{f_{\mathrm{PCC}-m}}^{2}} \cdot \hat{v}_{f_{\mathrm{PCC}-m}}=B_{c_{\mathrm{ref}}-m} \cdot \omega \hat{v}_{f_{\mathrm{PCC}-m}} \\
B_{c_{\mathrm{ref}}-m} & =\frac{Q_{c_{\text {ref }-m}}}{V_{f_{\mathrm{PCC}-m}}^{2}} \\
W_{f-m} & =\frac{Q_{c_{-} \mathrm{ref}-m}}{\omega} .
\end{aligned}
$$

2) Flexible Compensation of Unwanted Currents: MFGTIs connected to the local PCCs are responsible for compensation of PQ disturbances caused by local loads. Thus, the following compensation current reference is generated for each MFGTI [13]:

$$
i_{\text {comp_ref-m }}=i_{r m}\left(1-k_{r m}\right)+i_{v m}\left(1-k_{v m}\right)
$$

where " $m$ " is the number of MFGTIs composing the microgrid.

When the MFGTI is switched OFF, load conformity factors are equal to those of the local point of coupling. However, when the MFGTI injects the RES-generated power $\left(P_{\mathrm{DG} m}\right)$ simultaneously to the compensation for PQ disturbances, the load conformity factors $\left(\lambda_{Q m}\right.$ and $\left.\lambda_{D m}\right)$ must be adapted to the condition in which the active power through the grid $\left(P_{g m}\right)$ depends not only on the load $\left(P_{m}\right)$, but also on the local power source $\left(P_{g m}=P_{m}-P_{\mathrm{DG} m}\right)$ [32]. Note that $P_{g m}$ is indirectly calculated, i.e., the grid current is not measured in the real system.

This defines the conformity factors effectively measured in the coupling points $\left(\lambda_{g Q m}\right.$ and $\left.\lambda_{g D m}\right)$, which are calculated considering active power injection, without any compensation. Thus, the weighting factors for reactive current $k_{r m}$ and residual current $k_{v m}$ are calculated as (18) and (19), respectively. These equations list the factors indirectly gauged at PCC $\left(\lambda_{g Q m}\right.$ and $\left.\lambda_{g D m}\right)$ with the desired conformity factors for the grid side $\left(\lambda_{g Q \text { ref }-m}\right.$ and $\left.\lambda_{g D \_ \text {ref }-m}\right)$ [13]. Reference factors are sent from the control center to each MFGTI using an Ethernet network

$$
\begin{aligned}
& k_{r m}=\frac{\lambda_{g Q m}}{\lambda_{g Q \_ \text {ref }-m}} \sqrt{\frac{1-\lambda_{g Q \text { ref }-m}^{2}}{1-\lambda_{g Q m}^{2}}} \\
& k_{v m}=\frac{\lambda_{g D \_ \text {ref }-m}}{\lambda_{g D m}} \sqrt{\frac{1-\lambda_{g D m}^{2}}{1-\lambda_{g D \_ \text {ref }-m}^{2}}} .
\end{aligned}
$$

It is worth to mention that the range of $\lambda_{g D} \_$ref $-m$ values may vary between 0 and $\lambda_{g D m}$. When $\lambda_{g D \text { ref } \_m}=0$, the corresponding compensation coefficient is $k_{v m}=0$, resulting in full compensation of void current $i_{v m}$. On the other hand, when $\lambda_{g D \_ \text {ref }-m}=\lambda_{g D m}$, the void current is not compensated. Similarly, the reactive current is fully compensated when $\lambda_{g Q \text { ref }-m}=1\left(k_{r m}=0\right)$ and is not compensated when $\lambda_{g Q \text { _ref }-m}=\lambda_{g Q m}\left(k_{r m}=1\right)$. Partial compensation of reactive and void current is obtained for $\lambda_{g Q m}<\lambda_{g Q \text { _ref }-m}<1$ and $0<\lambda_{g D \_ \text {ref }-m}<\lambda_{g D m}$, respectively.

Thus, by combining (14) and (17), the references for coordinated compensation and additional reactive power injection can be generated. 


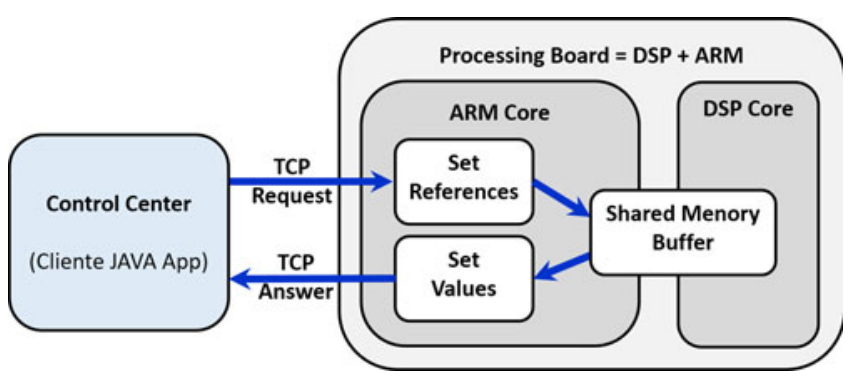

Fig. 5. General view of architecture used to control and supervise MFGTIs.

\section{B. Generation of the Reference Signal for Active Power Injection}

The injection of active power $\left(P_{\mathrm{DG} m}\right)$ generated by RES is achieved by synthesizing the following current reference:

$$
i_{\mathrm{DG} \_ \text {ref }-m}=\frac{\left\langle v_{\mathrm{DC} m}, i_{\mathrm{DC} m}\right\rangle}{V_{\mathrm{PCC}-m}^{2}} v_{\mathrm{PCC}-m}=\frac{P_{\mathrm{DG} m}}{V_{\mathrm{PCC}-m}^{2}} v_{\mathrm{PCC}-m}
$$

where

$i_{\text {DG_ref }-m}$ reference signal related to the power generated by the local RES;

$v_{\mathrm{DC} m} \quad$ MFGTI dc bus voltage;

$i_{\mathrm{DC} m} \quad$ DC from RES;

$v_{\mathrm{PCC} m} \quad$ MFGTI point of coupling voltage;

$V_{\mathrm{PCC}-m} \quad$ rms value of $v_{\mathrm{PCC}-m}$.

\section{Implementation of Synergistic Control and Coordinated Operation}

Figs. 1 and 4 show an overview of the control strategy and the diagram of a microgrid. The reference signal for each MFGTI is given by

$$
i_{F \_ \text {ref }-m}=i_{g P m}^{*}-i_{\text {DG_ref }-m}-i_{\text {comp_ref }-m}+i_{C \_ \text {ref }-m}
$$

where $i_{g P m}^{*}$ is the reference necessary to maintain constant $\mathrm{dc}$ bus voltage. This reference is generated by multiplying the normalized PCC voltage by the signal $I_{\mathrm{GP} m}$ produced by the $\mathrm{dc}$ bus voltage regulator. If the dc bus voltage tends to deviate from the set point ( $v_{\mathrm{DC} m}^{*}$ ), the $i_{g P m}$ is adjusted by the voltage controller, maintaining the $v_{\mathrm{DC} m}$ voltage regulated.

\section{Microgrid Remote Management Center}

The general scheme used for communication among the proposed control center and the remote MFGTIs is shown in Fig. 5. It is also possible to connect other devices to the center, such as TSC or any elements with communication capability.

The center allows commands and operation references to be sent to MFGTIs and to collect information about inverter operations, such as voltage level, active power, portions of processed power (such as reactive and residual power), rms current, etc. The references sent from the center to MFGTIs are, for example, the value of the desired voltage for the bus, compensation strategy and level of compensation, value for the quality indexes at the point of coupling, etc. It is possible to operate the MFGTIs with online adjustments, allowing for the use of complex coordination and cooperation strategies among MFGTIs.

The communication structure is based on the Ethernet $10 / 100 \mathrm{Mb} / \mathrm{s}$ standard. Despite the high throughput, the amount of data exchanged between the center and MFGTIs is exceptionally small. This is due to the fact that references for the MFGTI, as well as measurements monitored by the center, are obtained from the steady-state operation, with measures usually taken in one or more grid cycles (rms and average values as well as power calculation). Thus, the communication network latency does not affect data exchange.

In addition to the control center, this section also discusses the hardware/firmware system that, locally and digitally, controls each of the MFGTIs.

\section{A. MFGTI Local Control and Supervision Architecture}

Communication between the control center and MFGTIs is performed as shown in Fig. 5. Data exchange occurs following a client request (control center). Then, the MFGTI (server) sends the required information.

The system on chip (SoC) is composed of two asymmetric processing cores. The first core is a digital signal processor (DSP) with floating point arithmetic. The second core is a general purpose microcontroller from the ARM Cortex M4 series. The two cores exchange data through a shared RAM memory. The DSP core processes the control algorithms, conducts current decomposition, and calculates load conformity factors. The ARM core runs the DSP management program and communicates with a remote center via transmission control protocol/internet protocol (TCP/IP). User commands sent through the control center can remotely activate or deactivate MFGTIs, change operational mode, and adjust the references.

The main reason for choosing the TCP/IP over Ethernet protocol is, besides easy implementation, that the $\mathrm{SoC}$ TMS320F28M335 from Texas Instruments, used to perform the local control of MFGTIs, provides this protocol for communication. Thus, the communication interface already available in the development kit allowed us to speed up the development of a conceptual proof and the obtainment of experimental results.

Data exchange between the center and MFGTIs is implemented through type length value (TLV) data packages. TLV protocol simplicity facilitates the implementation of embedded systems. Furthermore, the total overhead information is extremely low. However, due to communication using TCP/IP protocol over Ethernet, the TLV package is encapsulated within a TCP/IP package that, in turn, is encapsulated in an Ethernettype package [38]-[40].

TCP/IP and Ethernet protocols are processed transparently in the application through software stacks that run both on the transmitting and receiving computers. Therefore, from the point of view of communication between the control center and the MFGTIs, only the TLV protocol is processed directly.

\section{B. Centralized Control and Monitoring Center}

Fig. 6 shows the home screen of the online control and monitoring center, developed using JavaScript [41]-[43]. The choice 


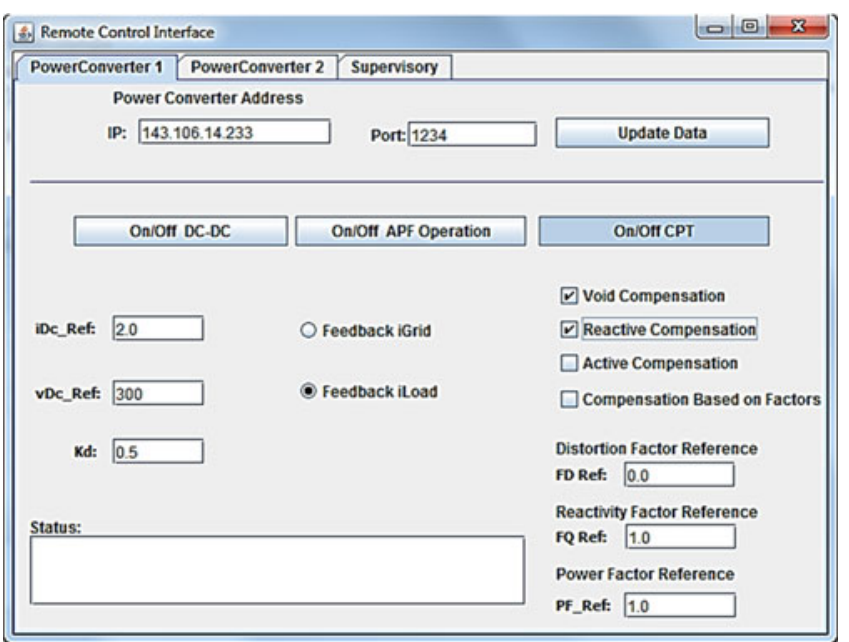

Fig. 6. Centralized control center screen for sending references and commands to the MFGTI.

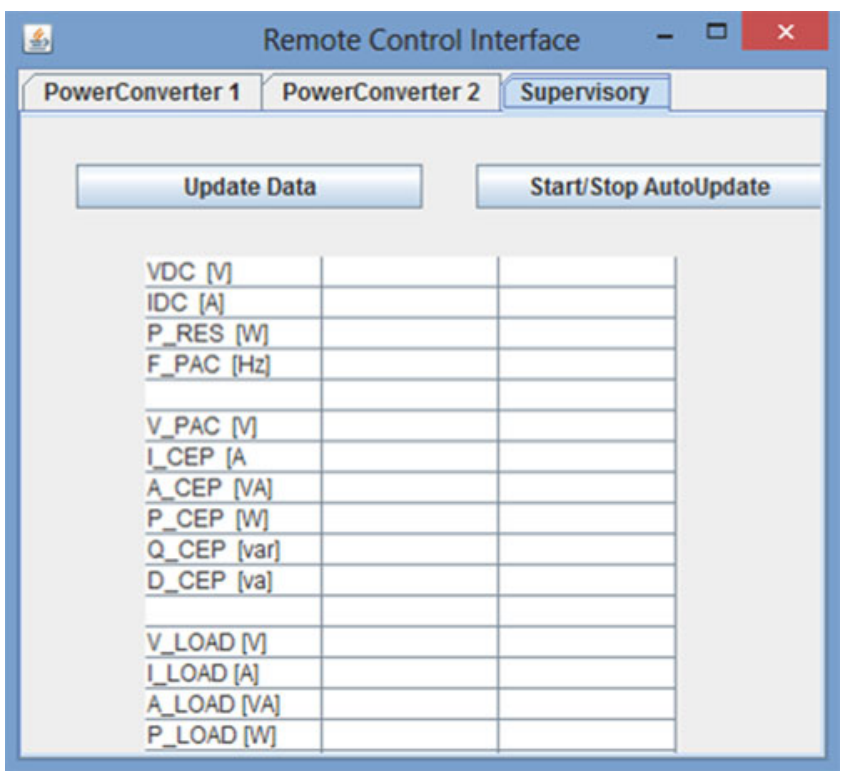

Fig. 7. Centralized control center screen for reading data from MFGTIs.

of this platform is basically due to the running of the software in the Java virtual machine, which can run on many operating systems. Furthermore, through the object orientation concept, it is only necessary to program a class to manage a single MFGTI as the same code can quickly be extended to manage other MFGTIs. Note that each MFGTI is identified by a unique IP address.

Fig. 7 shows the screen for reading information received from all operational MFGTIs. MFGTI information can be updated whenever the update button is activated or automatically updated (auto update button). The interval between the updates depends on the number of MFGTIs being monitored due to the polling scheme, in which the center requires and receives information from each of the MFGTIs sequentially. When the last MFGTI sends its information, the data forms are updated and the routine restarts.

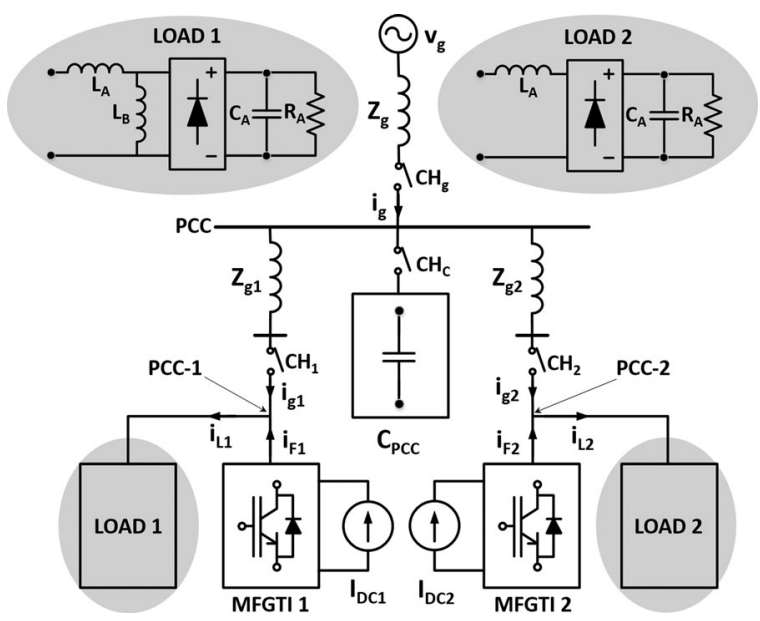

Fig. 8. Experimental microgrid with MFGTIs connected to the power grid.

TABLE II

SyStem PARAMETERS SHOWN IN FIG. 8

\begin{tabular}{lcc}
\hline \hline MFGTI 1 and MFGTI 2 & LCL filter & $L_{1}=L_{2}=0.5 \mathrm{mH}$ \\
& & $R_{1}=R_{2}=100 \mathrm{~m} \Omega$ \\
& & $C_{01}=C_{02}=3 \mu \mathrm{F}$ \\
& $R_{01}=R_{02}=10 \mathrm{~m} \Omega$ \\
& DC bus & $V_{D C 1}=V_{D C 2}=300 \mathrm{~V}$ \\
& $C_{D C 1}=C_{D C 2}=1 \mathrm{mF}$ \\
& RES & $I_{D C 1}=I_{D C 2}=3.25 \mathrm{~A}$ \\
\hline Grid & $Z_{g}$ & $R_{g}=10 \mathrm{~m} \Omega ; L_{g}=0.1 \mathrm{mH}$ \\
& $R_{g 1}=0.1 \Omega ; L_{g}=0.56 \mathrm{mH}$ \\
& $Z_{g 2}$ & $R_{g 2}=0.1 \Omega ; L_{g 2}=1 \mathrm{mH}$ \\
& Voltage/frequency & $V g=127 \mathrm{~V} ; f=60 \mathrm{~Hz}$ \\
& Voltage distortion & Total harmonic distortion \\
& & $(\mathrm{THD})_{V}=1.8 \%$ \\
\hline Loads & Load-1 & $L_{A}=2 \mathrm{mH}$ \\
& & $R_{A}=95 \Omega ; C_{A}=1000 \mu \mathrm{F}$ \\
& $L_{B}=70 \mathrm{mH} ; R_{B}=3 \Omega$ \\
& Load-2 & $L_{\mathrm{A}}=1 \mathrm{mH}$ \\
& & $R_{A}=100 \Omega ; C_{A}=1 \mathrm{mF}$ \\
\hline Reactive compensator & Capacitor & $C_{\mathrm{PCC}}=40 \mu \mathrm{F}$ \\
\hline \hline
\end{tabular}

Thus, the higher the number of MFGTIs, the longer the update interval. The polling time for a single MFGTI is approximately 2 s. For a hypothetical microgrid with 10 MFGTIs, the time necessary to collect the information from all MFGTIs would be $20 \mathrm{~s}$

\section{EXPERIMENTAL RESULTS}

The structure of the implemented microgrid is shown in Fig. 8. A local load with nonlinear characteristics is connected with each MFGTI. The topology is similar to a home, or a small commercial/industrial installation, including a range of local power sources. The arrows indicate the currents used to analyze the results. The main parameters for MFGTIs, electrical grid, and loads are listed in Table II. The possibility of coordination among MFGTIs is demonstrated by means of measurements taken at different points of the microgrid. Rated power (active and reactive) for Load-1 is $385 \mathrm{~W}$ and 600 var, and 


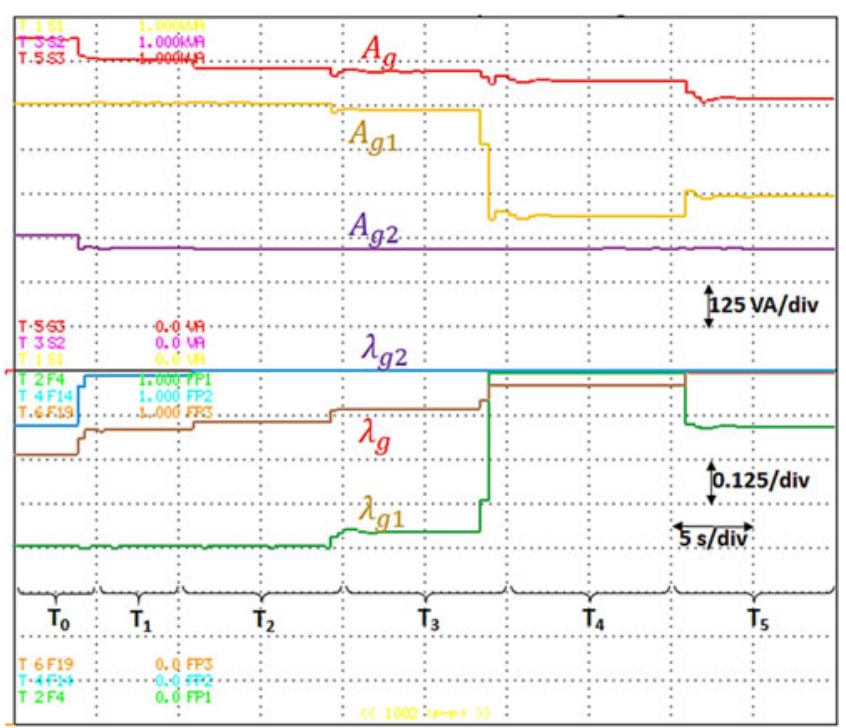

Fig. 9. Coordinated operation of MFGTIs and capacitor bank to improve the power factor at the point of coupling.

$330 \mathrm{~W}$ and 50 var for Load-2. The reactive power of the capacitor bank is approximately 250 var. When the capacitor is connected to the PCC, it compensates part of the load's reactive power.

\section{A. Coordinated Operation for Unwanted Currents Compensation}

In a first scenario, the main objective is to improve the PQ at the PCC by coordination among the MFGTIs considering the capacitor $\left(C_{\mathrm{PCC}}\right)$ is connected to the PCC. Commands are sent to MFGTIs to operate only as APFs. Each MFGTI will perform the local compensation of the harmonics generated by its own nonlinear load, through the adjustment of $\lambda_{g D \_ \text {ref }-m}$. Then, the compensation of the reactive power is shared among the MFGTIs, through the adjustment of reference $\lambda_{g Q \_ \text {ref }-m}$. As result of these actions, it is expected to achieve a near unitary power factor at the PCC.

The microgrid's operator sets the compensation references of each MFGTI using the remote control center through the following sequence of commands:

1) $T_{0}:$ MFGTI- 1 and MFGTI-2 disconnected (no compensation);

2) $T_{1}$ : MFGTI-2 compensates only for the harmonics of Load-2;

3) $T_{2}$ : MFGTI-2 performs full compensation of harmonics and reactive power of Load-2;

4) $T_{3}$ : MFGTI-2 performs full compensation of Load- 2 and MFGTI-1 compensates only for the harmonics of Load-1;

5) $T_{4}$ : MFGTI-1 and MFGTI-2 perform full compensation of their respective local loads; and

6) $T_{5}$ : MFGTI-2 performs full compensation of Load-2 and MFGTI-1 compensates for all the harmonics and partially for reactive power for Load-1;

Fig. 9 shows the apparent power evolution and the power factor gauged at the PCC for each MFGTIs $\left(A_{g 1}, A_{g 2}, \lambda_{g 1}\right.$, and $\left.\lambda_{g 2}\right)$, as well as in the PCC of the microgrid $\left(A_{q}\right.$ and $\left.\lambda_{q}\right)$. Fig. 10 shows voltage and currents waveforms at the PCCs during each interval.

Interval $T_{0}$ : As both MFGTIs are disconnected, the network supplies all the load power, including the distortions $\left(D_{L}\right.$ and $Q_{L}$ ). Initially, the currents in the PCCs are highly distorted and, consequentially, the power factors are low (see Fig. 10 and Table III).

Interval $T_{1}$ : As MFGTI-2 provides all the residual power required by Load-2 $\left(\lambda_{g D_{\text {ref }}-2}=0\right)$, the residual current (harmonics) originated by this load no longer circulates through the grid, and [as shown in Fig. 10(b)] current $i_{g 2}$ practically has a sine format. Looking at Table III, the power factor at PCC-2 was improved substantially. Load-2 (as shown in Fig. 8) is a single-phase rectifier with capacitive filter, which demands low reactive power. Thus, by compensating the residual power, responsible for almost all the distortion originating from this load, the power factor is close to the unit.

Interval $T_{2}$ [see Fig. 10(c)]: As MFGTI-2 maintains the compensation of residual power and the control center sends the order to compensate the reactive power $\left(\lambda_{g Q_{\text {_ref }-2}}=1\right)$, the power factor at PCC-2 becomes practically unitary $\left(\lambda_{g 2} \approx 1\right)$. As the reactive power of Load-2 is small, total compensation reaches practically the same result as the previous compensation (interval of $T_{1}$ ).

Interval $T_{3}$ : MFGTI-2 fully compensates Load-2 disturbances. MFGTI-1 is connected to PCC-1, and the order from the control center is to compensate the residual current from Load-1 $\left(\lambda_{g D \_ \text {ref }-1}=0\right)$. From Fig. 9 and Table III, a slight improvement in the power factor at PCC-1 $\left(\lambda_{g 1}\right)$ is seen, as the apparent power that circulates through PCC- 1 is reduced due to residual power compensation. Fig. 10(d) shows that harmonics compensation makes the current through PCC-1 $\left(i_{g 1}\right)$ similar to the voltage. The phase shift between voltage and current at PCC-1 is due to the reactive power of Load-1, which is not being compensated.

Interval $T_{4}$ : In this interval, MFGTI-2 is maintained in the same configuration. The control center sends a command to MFGTI-1 to add reactive power compensation $\left(\lambda_{g} Q_{\text {ref }-1}=1\right)$, thereby assuming full compensation of Load-1 disturbances. As shown in Fig. 10(e), the amplitude of the PCC-1 current reduces. The current $i_{g 1}$ presents a waveform identical to that of the voltage. However, due to the $C_{\mathrm{PCC}}$ capacitor, the current that circulates through the distribution network $\left(i_{g}\right)$ leads the voltage at PCC. Despite the microgrid being completely compensated $\left(\lambda_{g 1}=\lambda_{g 2}=1\right)$, the power factor seen at the point of coupling between the microgrid and distribution grid is not unitary $\left(\lambda_{g}=\right.$ 0.959 ).

Interval $T_{5}$ [see Fig. 10(f)]: To obtain the unity power factor at the PCC, the reactive power of Load-1 must be partially compensated by MFGTI-1. Thus, the control center adjusts the reactivity factor for PCC- 1 to $\lambda_{g} Q_{\text {_ref }-1}=0.86$. The action leads to a lower power factor at PCC-1, since around 245 var is no longer compensated by the MFGTI, as this value is already being injected by the $C_{\mathrm{PCC}}$ capacitor. However, the coordinated compensation of MFGTIs maximizes the PCC power factor $\left(\lambda_{g} \approx 0.998\right)$. 

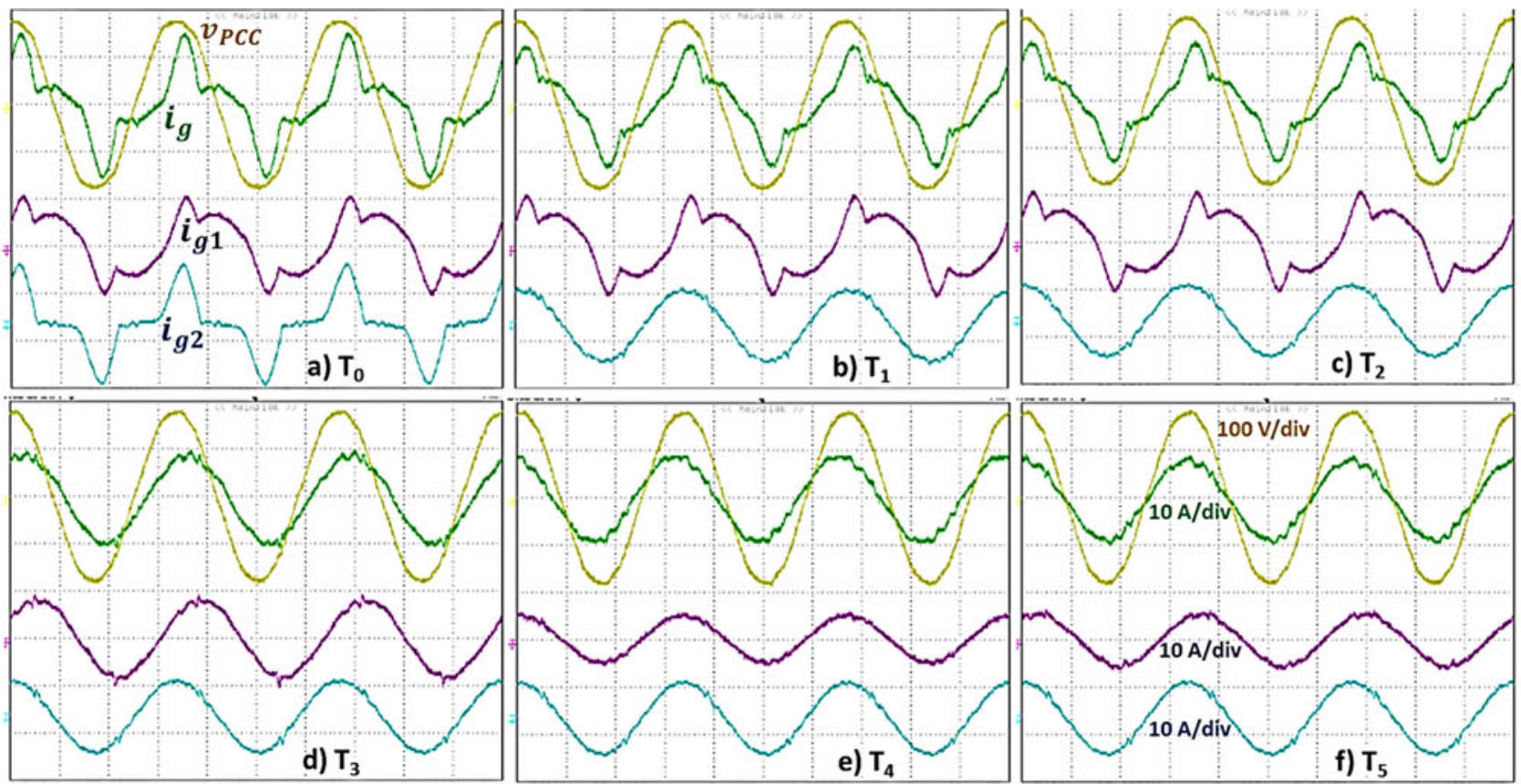

Fig. 10. Voltage and current waveforms during MFGTI coordinated unwanted currents compensation.

TABLE III

PCCS MEASUREMENTS DURING MFGTI COORDINATED COMPENSATION-MFGTIS OPERATING ONLY AS APF

\begin{tabular}{lcccccc}
\hline \hline Quantity & $T_{0}$ & $T_{1}$ & $T_{2}$ & $T_{3}$ & $T_{4}$ & $T_{5}$ \\
\hline$A_{g}$ & 943 & 885 & 863 & 851 & 830 & 780 \\
$A_{g 1}$ & 756 & 758 & 759 & 739 & 449 & 495 \\
$A_{g 2}$ & 383 & 343 & 341 & 342 & 343 & 343 \\
$P_{g}$ & 724 & 737 & 740 & 763 & 797 & 778 \\
$P_{g 1}$ & 388 & 388 & 387 & 411 & 448 & 429 \\
$P_{g 2}$ & 325 & 339 & 341 & 342 & 343 & 343 \\
$Q_{g}$ & 417 & 420 & 367 & 373 & -229 & 0 \\
$Q_{g 1}$ & 606 & 607 & 608 & 613 & 16 & 245 \\
$Q_{g 2}$ & 53 & 54 & 0 & 0 & 0 & 0 \\
$\lambda_{g}$ & 0.767 & 0.833 & 0.858 & 0.897 & 0.959 & 0.998 \\
$\lambda_{g 1}$ & 0.512 & 0.512 & 0.512 & 0.613 & 0.998 & 0.866 \\
$\lambda_{g 2}$ & 0.847 & 0.986 & 0.999 & 0.999 & 0.999 & 0.999 \\
THD $_{I g}$ & 38 & 30 & 30 & 5.0 & 5.2 & 5.8 \\
THD $_{I g 1}$ & 31 & 31.7 & 31.6 & 4.8 & 5.0 & 5.0 \\
THD $_{I g 1}$ & 57 & 5.4 & 5.5 & 5.8 & 5.4 & 4.4 \\
\hline \hline
\end{tabular}

Discussion: The reference value $\left(\lambda_{g} Q_{\text {ref } \_1}=0.86\right)$ that leads to a null reactive power at the PCC is calculated through (22), which defines the reactivity factor

$$
\lambda_{g Q 1}=P_{g 1} / \sqrt{P_{g 1}^{2}+Q_{g 1}^{2}} .
$$

In this case, as the whole residual power is being compensated by MFGTI-1, only the reactive power affects the power factor at PCC-1, then $\lambda_{g 1}=\lambda_{g Q 1}$. The reactivity factor is calculated using the value of the active power at the point of coupling of MFGTI $\left(P_{g 1}\right)$. Initially, the value of the reactive power is taken throughout the PCC after the full compensation of the microgrid (interval $\mathrm{T}_{4}$ ). Certain iterations are necessary to attain the desired value for reactive power. As the MFGTI no longer compensates part of the load's reactive power, the voltage drop at
PCC-1 impedance reduces the rms voltage, which decreases the load active power. At the end of the process, a power factor of $\left(\lambda_{g 1}=\lambda_{g Q 1}=0.86\right)$ is obtained, resulting in $P_{g 1}=429 \mathrm{~W}$ and $Q_{g 1}=245$ var. It is worth to mention that the microgrid's operator performs the iterations manually by setting the reference $\lambda_{g Q_{\text {_ref_l }}}$ in the control center until the reactive power measured at the PCC becomes zero.

This case shows that the coordinated compensation strategy can be used to improve PQ indicators at any point of the microgrid. Adjustment flexibility for load conformity factors (reactivity and distortion) facilitates the implementation of coordinated compensation with other types of compensators, allowing the desired values to be obtained for the range of PQ indexes.

Thus, the scenario shown above is coherent from a practical point of view, as harmonics are compensated by MFGTIs, whereas the reactive power is compensated by the capacitive bank. Furthermore, MFGTIs may complement the reactive power compensation to obtain a microgrid with resistive behavior, providing a high power factor and low harmonic distortion.

\section{B. Coordinated Operation for Unwanted Currents Compensation and Active Power Injection With Equalization of the Apparent Power of MFGTIs}

This section discusses the coordination of MFGTIs operating as multifunctional devices. Each MFGTI, besides injecting the active power generated by the RES, also fulfills the role of compensating reactive power and harmonic distortion due to local loads.

The goal is to improve the PQ in the PCC and distribute the compensation efforts among MFGTIs to balance the apparent power processed by each converter. The $C_{\mathrm{PCC}}$ capacitor, when connected to the PCC of the microgrid, cooperates partially 

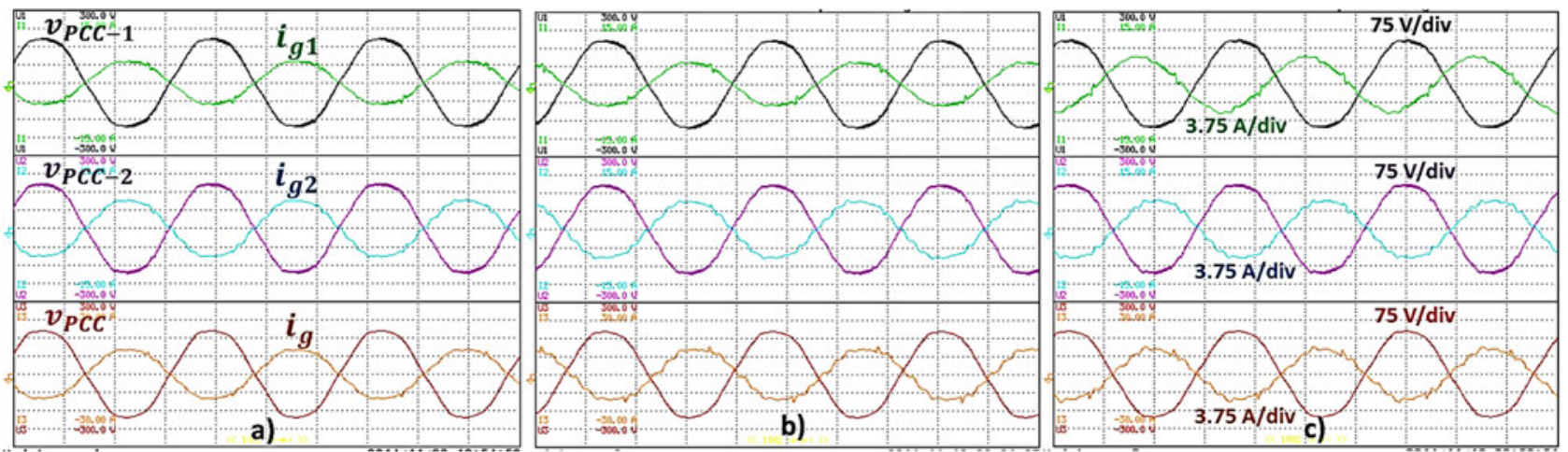

Fig. 11. Waveforms at common points of coupling: (a) total compensation without the capacitor, (b) total compensation with the capacitor, and (c) coordinated compensation.

TABLE IV

PCCS MEASUREMENTS DURING MFGTIS COORDINATED OPERATION FOR UNWANTED CURRENTS COMPENSATION AND ACTIVE POWER INJECTION

\begin{tabular}{|c|c|c|c|}
\hline Quantity & $\begin{array}{l}\text { Without } \\
\text { capacitor }\end{array}$ & $\begin{array}{l}\text { With } \\
\text { capacitor }\end{array}$ & $\begin{array}{l}\text { With capacitor } \\
\text { coordinated }\end{array}$ \\
\hline$\lambda_{g} D \_$ref -1 & 0.0 & 0.0 & 0.0 \\
\hline$\lambda_{g} Q_{\text {_ref }-1}$ & 1.0 & 1.0 & 0.84 \\
\hline$Q_{c \_ \text {ref }-1}[\mathrm{var}]$ & 0.0 & 0.0 & 0.0 \\
\hline$\lambda_{g D \_ \text {ref }-2}$ & 0.0 & 0.0 & 0.0 \\
\hline$\lambda_{g Q \text { _ref }-2}$ & 1.0 & 1.0 & 1.0 \\
\hline$Q_{c_{\text {_ref-2 }}}[\mathrm{var}]$ & 0.0 & 0.0 & 50.0 \\
\hline$A_{g 1}[\mathrm{VA}]$ & 427 & 427 & 532 \\
\hline$A_{q 2}[\mathrm{VA}]$ & 560 & 560 & 564 \\
\hline$A_{q}[\mathrm{VA}]$ & 978 & 976 & 999 \\
\hline$P_{g 1}[\mathrm{~W}]$ & -426 & -427 & -446 \\
\hline$P_{q 2}[\mathrm{~W}]$ & -560 & -559 & -561 \\
\hline$P_{g}[\mathrm{~W}]$ & -978 & -976 & -995 \\
\hline$Q_{q 1}$ [var] & -4 & -4 & 288 \\
\hline$Q_{q 2}[\mathrm{var}]$ & -4 & -3 & -54 \\
\hline$Q_{g}[\operatorname{var}]$ & 1 & -255 & -10 \\
\hline$\lambda_{g 1}$ & -0.999 & -0.999 & -0.838 \\
\hline$\lambda_{g 2}$ & -0.999 & -0.999 & -0.994 \\
\hline$\lambda_{q}$ & -0.999 & -0.965 & -0.999 \\
\hline $\mathrm{THD}_{i g 1}$ & 5.4 & 5.7 & 6.1 \\
\hline THD $_{i g 2}$ & 5.7 & 6.1 & 6.1 \\
\hline $\mathrm{THD}_{\mathrm{ig}}$ & 5.0 & 8.3 & 8.5 \\
\hline $\mathrm{THD}_{v} \mathrm{PCC} 1$ & 4.0 & 3.8 & 3.8 \\
\hline $\mathrm{THD}_{v} \mathrm{PCC}_{2}$ & 4.1 & 4.0 & 3.9 \\
\hline $\mathrm{THD}_{v}$ PCC & 4.0 & 3.7 & 3.7 \\
\hline$A_{\text {MFGTI_1 }}[\mathrm{VA}]$ & 1035 & 1035 & 895 \\
\hline$A_{\mathrm{MFGTI} 2}$ [VA] & 905 & 902 & 910 \\
\hline
\end{tabular}

with reactive power compensation. In this analysis, MFGTI-1 provides around $850 \mathrm{~W}$ to PCC-1, whereas MFGTI-2 provides around $900 \mathrm{~W}$ to PCC-2. Fig. 11 shows waveforms for voltage and current at different points of the microgrid (PCC-1, PCC-2, and PCC) for three different conditions.

Initially, $C_{\mathrm{PCC}}$ is not connected and both MFGTIs provide complete compensation for disturbances caused by the respective local loads. As expected, in Fig. 11(a), the currents at the PCCs of the microgrid have the waveform of their respective voltages. The counter phase indicates the injection of power into the grid. As shown in Table IV, the apparent power processed by MFGTI-1 is 1035 VA, whereas MFGTI-2 processes approximately 905 VA.
Hypothetically, if the nominal power of the MFGTIs was 900 VA, MFGTI-1 violates its power rating. The insertion of $C_{\mathrm{PCC}}$ aims to reduce the amount of reactive power processed by MFGTIs, as this level of power cannot be maintained for a long time.

Table IV shows that the insertion of the capacitor $\left(C_{\mathrm{PCC}}\right)$ has practically no effect on the PQ indicators at PCC-1 and PCC-2, nor does it affect the apparent power processed by MFGTIs, as the compensation references for the converters were not altered.

However, since the reactance of $C_{\mathrm{PCC}}$ at harmonic frequencies is lower than at the fundamental, the harmonics in the PCC voltage, even with low amplitude, cause high harmonic currents through the grid and the capacitor. Fig. 11(b) shows a slight distortion in the current through the grid $\left(i_{g}\right)$. Note further that the power factor at PCC is not unitary, $\lambda_{g}=-0.965$. The negative power factor signal indicates that the reactive power provided by the capacitor is being injected into the grid. To achieve unitary power factor, it is necessary to absorb the reactive power from the capacitor. This action is presented in Fig. 11(c), showing the coordinated operation of MFGTIs.

The microgrid absorbs the reactive power, around 250 var, coming from the $C_{\mathrm{PCC}}$. Therefore, MFGTI-1, which compensated around 600 var required by Load-1, drops to compensating at just 300 var. MFGTI-2 fully compensates the reactive power demanded by Load- 2 and injects around an additional 50 var into PCC-2. The pair of MFGTIs supply approximately 350 var of the total 600 var required by Load- 1 . The supplementary reactive power required by Load- 1 is achieved by means of the $C_{\text {PCC }}$ capacitor, which injects 250 var. The additional reactive power injected by MFGTI-2 is achieved by sending reactive power reference from the center to the MFGTI-2.

At the end, the phase shift between voltage and current at the PCC is practically zero. The power factor measured at the PCC again becomes almost unitary, $\lambda_{g} \approx 1$. Fig. 12 indicates the reactive power flow circulating through the microgrid and Table IV sums up the main PQ parameters for each of the three situations described above.

The capacitor connection aligned with the coordinated operation of MFGTIs led to the reduction and balance of their efforts (processed apparent power). Following coordinated compensation, MFGTIs begin working close to the 


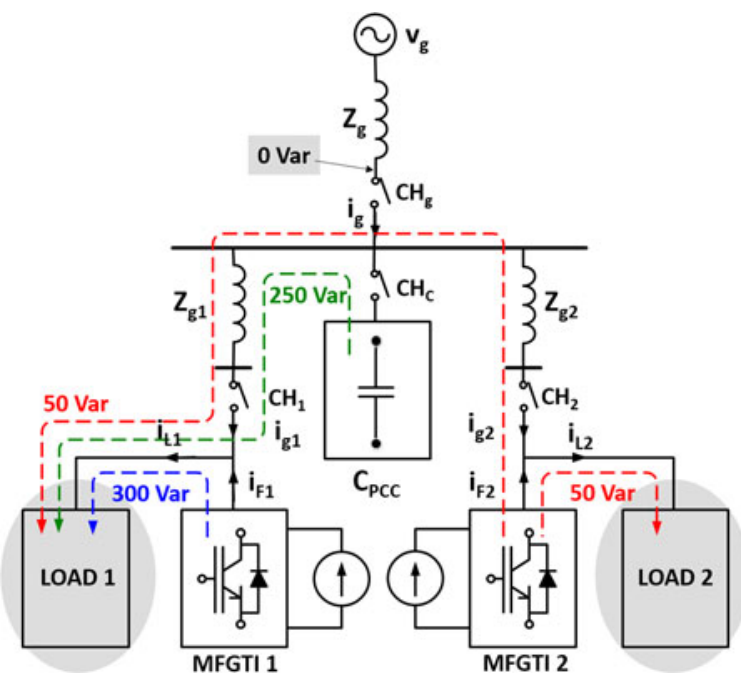

Fig. 12. Flow of reactive power through coordinated operation between MFGTIs and $C_{\mathrm{PCC}}$.

rated power $\left(A_{\mathrm{MFGTI}-1}=895 \mathrm{VA}\right.$ and $\left.A_{\mathrm{MFGTI}-2}=910 \mathrm{VA}\right)$. With the reduction of the apparent power and current through MFGTIs, it is possible to use the converter capacities to inject more energy, since the local source has capacity for greater production.

\section{CONCLUSION}

Based on experimental results, our study showed that coordinated operation among MFGTIs and other compensation devices can contribute to reductions in the power and current processed by MFGTIs, maintaining coherent values for PQ indicators.

Furthermore, flexibility in adjusting the current references for disturbances compensation is fundamental to the cooperation among compensating devices. Through the control center, it was possible to coordinate operation to test the maximum MFGTI functionalities without surpassing the capacity of the converters. Depending on the profile of the local load connected to the MFGTI point of coupling, as well as the generation profile for each local power source, the installation of $\mathrm{PQ}$ compensation devices can thus be postponed. In other words, devices with lower per kVA costs can be installed, such as capacitor banks and thyristor controlled reactors (TCRs), which can cooperate with the MFGTIs already installed to obtain adequate PQ indicator values at the PCC.

\section{REFERENCES}

[1] Renewable Energy News and Information, Nashua, NH, USA, 2017. [Online]. Available: http://www.renewableenergyworld.com/index.html. Accessed on: Jan. 2017.

[2] J. M. Carrasco et al., "Power electronic systems for the grid integration of renewable energy sources: A survey," IEEE Trans. Ind. Electron., vol. 53, no. 4, pp. 1002-1016, Jun. 2006, doi: 10.1109/TIE.2006.878356.

[3] A. Ipakchi and F. Albuyeh, "Grid of the future," IEEE Power Energy Mag., vol. 7, no. 2, pp. 52-62, Mar. 2009, doi: 10.1109/MPE.2008.931384.

[4] H. Farhangi, "The path of the smart grid," IEEE Power Energy Mag., vol. 8, no. 1, pp. 18-28, Jan. 2010, doi: 10.1109/MPE.2009.934876.
[5] E. Romero-Cadaval, B. Francois, M. Malinowski, and Q.-C. Zhong, "Gridconnected photovoltaic plants: An alternative energy source, replacing conventional sources," IEEE Ind. Electron. Mag., vol. 9, no. 1, pp. 18-32, Mar. 2015, doi: 10.1109/MIE.2014.2362211.

[6] V. C. Güngör et al., "Smart grid technologies: Communication technologies and standards," IEEE Trans. Ind. Informat., vol. 7, no. 4, pp. 529-539, Nov. 2011, doi: 10.1109/TII.2011.2166794.

[7] V. Cagri Gungor et al., "A survey on smart grid potential applications and communication requirements," IEEE Trans. Ind. Informat. vol. 9, no. 1, pp. 28-42, Feb. 2013, doi: 10.1109/TII.2012.2218253.

[8] N. R. Watson, T. L. Scott, and S. J. J. Hirsch, "Implications for distribution networks of high penetration of compact fluorescent lamps," IEEE Trans. Power Del., vol. 24, no. 3, pp. 1521-1528, Jul. 2009, doi: 10.1109/TPWRD.2009.2014036

[9] R. A. Mastromauro, M. Liserre, T. Kerekes, and A. Dell'Aquila, "A singlephase voltage-controlled grid-connected photovoltaic system with power quality conditioner functionality," IEEE Trans. Ind. Electron., vol. 56, no. 11, pp. 4436-4444, Nov. 2009, doi: 10.1109/TIE.2008.2004383.

[10] D. Li and Z. Q. Zhu, "A novel integrated power quality controller for microgrid," IEEE Trans. Ind. Electron., vol. 62, no. 5, pp. 2848-2858, May 2015, doi: 10.1109/TIE.2014.2362495.

[11] F. P. Marafão, D. Brandao, A. Costabeber, and H. H. M. Paredes, "Multitask control strategy for grid-tied inverters based on conservative power theory," IET Renewable Power Gener., vol. 9, pp. 154-165, 2015, doi: 10.1049/iet-rpg.2014.0065.

[12] S. Munir and Y. W. Li, "Residential distribution system harmonic compensation using PV interfacing inverter," IEEE Trans. Smart Grid, vol. 4, no. 2, pp. 816-827, Jun. 2013, doi: 10.1109/TSG.2013.2238262.

[13] J. P. Bonaldo, H. K. Morales Paredes, and J. A. Pomilio, "Control of single-phase power converters connected to low-voltage distorted power systems with variable compensation objectives," IEEE Trans. Power Electron., vol. 31, no. 3, pp. 2039-2052, Mar. 2016, doi: 10.1109/TPEL.2015.2440211.

[14] J. He, Y. W. Li, F. Blaabjerd, and X. Wang, "Active harmonic filtering using current-controlled, grid-connected DG units with closed-loop power control," IEEE Trans. Power Electron., vol. 29, no. 2, pp. 642-653, Feb. 2014, doi: 10.1109/TPEL.2013.2255895.

[15] J. He, Y. W. Li, and M. S. Munir, "A flexible harmonic control approach through voltage controlled DG-grid interfacing converters," IEEE Trans. Ind. Electron., vol. 59, no. 1, pp. 444-455, Jan. 2012, doi: 10.1109/TIE.2011.2141098.

[16] J. P. Bonaldo, H. K. M. Paredes, A. Costabeber, and J. A. Pomilio, "Adaptive saturation system for grid-tied inverters in low voltage residential micro-grids," in Proc. Int. Conf. Environ. Elect. Eng., 2015, pp. 784-789, doi: 10.1109/EEEIC.2015.7165264.

[17] H. Akagi, "Control strategy and site selection of a shunt active filter for damping of harmonic propagation in power distribution systems," IEEE Trans. Power Del., vol. 12, no. 1, pp. 354-363, Jan. 1997, doi: $10.1109 / 61.568259$.

[18] P. Jintakosonwit, H. Fujita, H. Akagi, and S. Ogasawara, "Implementation and performance of cooperative control of shunt active filters for harmonic damping throughout a power distribution system," IEEE Trans. Ind. Appl., vol. 39, no. 2, pp. 556-564, Mar./Apr. 2003, doi: 10.1109/TIA.2003.808959.

[19] P. T. Cheng and T. L. Lee, "Distributed active filter systems (DAFS): A new approach to power systems harmonics," IEEE Trans. Ind. Appl., vol. 42, no. 5, pp. 1301-1309, Sep./Oct. 2006, doi: 10.1109/ TIA.2006.880856.

[20] P. T. Cheng and T. L. Lee, "Analysis of harmonic damping effect of the distributed active filter system," IEEJ Trans. Ind. Appl., vol. 126, pp. 605614, May 2006, doi: 10.1541/ieejias.126.605.

[21] T.-L. Lee, P.-T. Cheng, H. Akagi, and H. Fujita, "A dynamic tuning method for distributed active filter systems," IEEE Trans. Ind. Appl., vol. 44, no. 2 , pp. 612-623, Mar./Apr. 2008, doi: 10.1109/TIA.2008.916596.

[22] E. Tedeschi and P. Tenti, "Cooperative design and control of distributed harmonic and reactive compensators," Przeglad Elektrotechniczny (Elect. Rev.), vol. 84, no. 6, pp. 23-27, Jun. 2008.

[23] H. K. M. Paredes, A. Costabeber, and P. Tenti, "Application of conservative power theory to cooperative control of distributed compensators in smart grids," Przeglad Elektrotechniczny (Elect. Rev.), vol. 87, no. 1, pp. 1-7, 2011.

[24] K. T. Tan, P. L. So, Y. C. Chu, and M. Z. Q. Chen, "Coordinated control and energy management of distributed generation inverters in a microgrid," IEEE Trans. Power Del., vol. 28, no. 2, pp. 704-713, Apr. 2013, doi: 10.1109/TPWRD.2013.2242495 
[25] X. Liu, P. Wang, and P. C. Loh, "A hybrid AC/DC microgrid and its coordination control," IEEE Trans. Smart Grid, vol. 2, no. 2, pp. 278-286, Jun. 2011, doi: 10.1109/TSG.2011.2116162.

[26] J. Han, S. K. Solanki, and J. Solanki, "Coordinated predictive control of a wind/battery microgrid system," IEEE J. Emerg. Sel. Topics Power Electron., vol. 1, no. 4, pp. 296-305, Dec. 2013, doi: 10.1109/JESTPE.2013.2282601.

[27] M. Savaghebi, A. Jalilian, J. C. Vasquez, and J. M. Guerrero, "Secondary control for voltage quality enhancement in microgrids," IEEE Trans. Smart Grid, vol. 3, no. 4, pp. 1893-1902, Dec. 2012, doi: 10.1109/TSG.2012.2205281.

[28] A. Mortezaei, M. Simoes, M. Savaghebi, J. Guerrero, and A. AlDurra, "Cooperative control of multi-master-slave islanded microgrid with power quality enhancement based on conservative power theory," IEEE Trans. Smart Grid, vol. 99, pp. 1-12, 2016, to be published. doi: 10.1109/TSG.2016.2623673.

[29] P. Tenti, H. K. M. Paredes, and P. Mattavelli, "Conservative power theory, a framework to approach control and accountability issues in smart microgrids," IEEE Trans. Power Electron., vol. 26, no. 3, pp. 664-673, Mar. 2011, doi: 10.1109/TPEL.2010.2093153.

[30] D. I. Brandao, H. Guillardi, H. K. Morales-Paredes, F. P. Marafão, and J. A. Pomilio, "Optimized compensation of unwanted current terms by AC power converters under generic voltage conditions," IEEE Trans. Ind. Electron., vol. 63, no. 12, pp. 7743-7753, Dec. 2016, doi: 10.1109/TIE.2016.2594226.

[31] H. K. M. Paredes, F. P. Marafão, P. Mattavelli, and P. Tenti, “Application of conservative power theory to load and line characterization and revenue metering," in Proc. IEEE Appl. Meas. Power Syst., Sep. 2012, pp. 1-6, doi: 10.1109/AMPS.2012.6343993.

[32] J. A. Pomilio, J. P. Bonaldo, H. K. Morales-Paredes, and P. Tenti, "About power factor and THDi in the smart micro-grid scenario," in Proc. IEEE Brazilian Power Electron. Conf. 1st Southern Power Electron. Conf., 2015, pp. 1-5, doi: 10.1109/COBEP.2015.7420183.

[33] Y. Tang, P. C. Loh, P. Wang, F. H. Choo, F. Gao, and F. Blaabjerg, "Generalized design of high performance shunt active power filter with output LCL filter," IEEE Trans. Ind. Electron., vol. 59, no. 3, pp. 1443-1452, Mar. 2012.

[34] I. J. Gabe, V. F. Montagner, and H. Pinheiro, "Design and implementation of a robust current controller for VSI connected to the grid through an LCL filter," IEEE Trans. Power Electron., vol. 24, no. 6, pp. 1444-1452, Jun. 2009, doi: 10.1109/TPEL.2009.2016097.

[35] P. Mattavelli and S. Buso, Digital Control in Power Electronics, 1st ed. San Rafael, CA, USA: Morgan \& Claypool Publ., 2006.

[36] A. Hasanzadeh, O. C. Onar, H. Mokhtari, and A. Khaligh, "A proportionalresonant controller-based wireless control strategy with a reduced number of sensors for parallel-operated UPSs," IEEE Trans. Power Del., vol. 25, no. 1, pp. 468-478, Jan. 2010, doi: 10.1109/TPWRD.2009.2034911.

[37] M. Karimi-Ghartemani, S. A. Khajehoddin, P. Jain, and A. Bakhshai, "A systematic approach to DC-bus control design in single-phase gridconnected renewable converters," IEEE Trans. Power Electron., vol. 28, no. 7, pp. 3158-3166, Jul. 2013.

[38] W. R. Stevens, The Protocols (TCP/IP Illustrated, Volume 1), 1st ed. Reading, MA, USA: Addison-Wesley, Dec. 31, 1993.

[39] A. S. Tanenbaum, Computer Networks, 4th ed. Englewood Cliffs, NJ, USA: Prentice-Hall, Aug. 2002.

[40] W. R. Stevens, B. Fenner, and A. M. Rudoff, Unix Network Programming, Volume 1: The Sockets Networking API, 3rd ed. Reading, MA, USA: Addison-Wesley, Nov. 2003.

[41] P. J. Deital and H. M. Deitel, "Java-How to Program," $8^{\text {th }}$ ed., London, U.K.: Pearson, 2009.

[42] C. S. Horstmann and G. Cornell, Core Java: Volume I-Fundamentals, 9th ed. Englewood Cliffs, NJ, USA: Prentice-Hall, Dec. 2012.

[43] C. S. Horstmann and G. Cornell, Core Java: Volume II-Advanced Features, 9th ed. Englewood Cliffs, NJ, USA: Prentice-Hall, Mar. 2013.

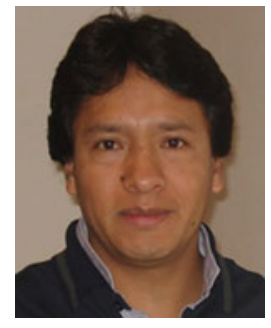

Helmo Kelis Morales-Paredes (S'10-M'11) received the B.S. degree from the National University of Saint Agustine, Arequipa, Peru, in 2002, and the M.Sc. and Ph.D. degrees from the University of Campinas, Campinas, Brazil, in 2006 and 2011, respectively, all in electrical engineering.

In 2009, he joined the Power Electronics Group, University of Padova, Padua, Italy, as a Visiting Student. In 2014, he joined the PEMC Group, University of Nottingham, Nottingham, U.K., as a Visiting Scholar. Since December 2011, he has been an Assistant Professor with São Paulo State University (UNESP), Sorocaba, Brazil, where he is the leader of the Group of Automation and Integrating Systems. His research interests include power quality, power theory, and power electronics applied to power systems.

Dr. Morales-Paredes is a member of the Brazilian Power Electronics Society and the Brazilian Automation Society. He was the recipient of a Prize Paper Award from the IEEE TRANSACTIONS ON POWER ELECTRONICS in 2011.

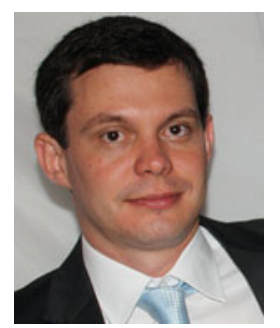

Jakson Paulo Bonaldo was born in Rosario Oeste, Brazil, in 1985. He received the B.S. degree from the Federal University of Mato Grosso, Cuiaba, Brazil, in 2008, and the M.Sc. and Ph.D. degrees from the University of Campinas, Campinas, Brazil, in 2010 and 2015, respectively, all in electrical engineering.

From 2010 to 2011, he was with the Padtec Optical Components and Systems, as a Firmware Engineer. Since 2013, he has been a Professor with the Department of Electronics, Federal University of Technology—Paraná, Campo Mourão, Brazil. His research interests include power quality, digital control, and renewable energy.

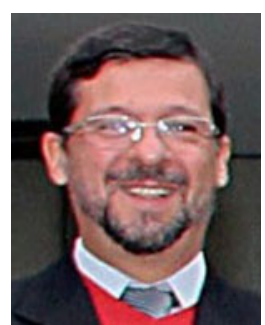

José Antenor Pomilio (M'92-SM'02) was born in Brazil, in 1960. He received the B.S., M.S., and Ph.D. degrees in electrical engineering from the University of Campinas, Campinas, Brazil, in 1983, 1986, and 1991, respectively.

From 1988 to 1991 , he was the Head of the Power Electronics Group, Brazilian Synchrotron Light Laboratory. He was a Visiting Professor with the University of Padova, Padua, Italy, in 1993 and 2015, and with the Third University of Rome, Rome, Italy, in 2003. He is currently a Professor with the School of Electrical and Computer Engineering, University of Campinas, where he has been teaching since 1984. His research interests include power electronics and power quality.

Dr. Pomilio was the President of the Brazilian Power Electronics Society during 2000-2002 and a member of the Administrative Committee of the IEEE Power Electronics Society during 1997-2002. He is currently an Associate Editor for the IEEE TRANSACTIONS ON POWER ELECTRONICS. 